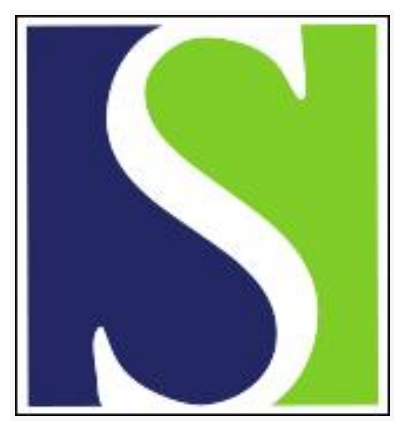

Scand J Work Environ Health 2001;27(1):1-4

https://doi.org/10.5271/sjweh.580

Issue date: Feb 2001

How confident can we be that acrylonitrile is not a human carcinogen?

by Hagmar L

Affiliation: Department of Occupational and Environmental Medicine, Lund University Hospital, SE-22185 Lund, Sweden. lars.hagmar@ymed.lu.se

Refers to the following texts of the Journal: $1998 ; 24$ suppl $2: 81-82$ 1998;24 suppl 2:71-80 1998;24 suppl 2:63-70 1998;24 suppl 2:54-62 1998;24 suppl 2:42-53 1998;24 suppl 2:25-41 1998;24 suppl 2:17-24 1998;24 suppl 2:10-16 1998;24 suppl 2:5-9 1998;24 suppl 2:3-4

Key terms: acrylonitrile; editorial; human carcinogen

This article in PubMed: www.ncbi.nlm.nih.gov/pubmed/11266141

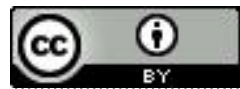




\section{How confident can we be that acrylonitrile is not a human carcinogen?}

Acrylonitrile is an important industrial chemical used in large quantities for, for example, the production of acrylic fibers (1). More than 100000 workers in the United States and Europe are potentially exposed. Whether occupational exposure to acrylonitrile causes increased risk for cancer in humans has been under debate for a long time. The proceedings from a workshop on this matter were recently published as a supplement to the Scandinavian Journal of Work Environment \& Health (2). The reason for the debate has been the apparent contradiction between experimental animal data and epidemiologic results. Two papers in this issue of the Scandinavian Journal of Work Environment \& Health add interesting information to this discussion $(3,4)$.

Animal experiments clearly show that acrylonitrile is carcinogenic (1). A high incidence of astrocytomas in the brain and spinal cord was the most consistent finding. In a 2-year inhalation study (6 hours/ day, 5 days/week) on rats, the lowest dose associated with an increased incidence of astrocytomas was $20 \mathrm{ppm}$ (5). Acrylonitrile can readily be taken up in the body by inhalation and metabolized to a reactive epoxide, cyanoethylene oxide, mainly by the cytochrome P450 isozyme CYP2E1 (6). Oyanoethylene oxide, which is thought to play an important role in the carcinogenic properties of acrylonitrile, is detoxified by glutathione conjugation in rodents, whereas in humans detoxification via epoxide hydrolase plays an important role. Hemoglobin adducts of acrylonitrile can be used as toxicologically relevant biomarkers of exposure (7).

Acrylonitrile has been the subject of a large number of epidemiologic studies of workers. Three large cohort studies provided exposure estimates for acrylonitrile (8-10). Ourrent operations in the production of acrylonitrile have exposure levels of $\leq 0.5 \mathrm{ppm}$ for an 8-hour time-weighted average, whereas past exposures have sometimes exceeded 20 ppm (11). A meta-analysis of 25 studies of acrylonitrileworkers indicated essentially null findings for lung, brain, and prostate cancer. In the cohort study by the National Cancer Institute (NCl) and the National Institute for Occupational Safety and Health (NIOSH), Poisson regression analyses showed, however, an excess of lung cancer (relative risk 1.5) in the highest quintile of cumulative exposure for acrylonitrile (8).

In this issue Marsh et al (3) present a reanalysis of the NCI-NIOSH cohort, for which standardized mortality ratios (SMR) are calculated for workers exposed and unexposed to acrylonitrile using national and regional comparison rates for the general population in the United States. The SMR value for lung cancer in the quintile of the most highly exposed workers was only 0.9 . With the use of external rates, the lower risk estimate was due to a significant 30\% deficit in lung cancer deaths for the unexposed workers. No plausible reason for this deficit is proposed. The authors of the original publication were very cautious in their interpretation of the data: " . . . analyses of exposure-response do not provide strong or consistent evidence for a causal association [p 25]" (8). The result of the reanalysis supports their cautiousness.

The International Agency for Research on Cancer (IARC) has recently changed its evaluation of acrylonitrile from probably $(2 \mathrm{~A})$ to possibly (2B) carcinogenic, due to the fact that there is inadequate evidence for carcinogenicity in humans, despite sufficient evidence with respect to experimental animals (1). According to the paper by Schulz et al in this issue, the cancer potency of acrylonitrile in the central nervous system (CNS), estimated from the best available animal inhalation study, is not inconsistent with observed deaths in the epidemiologic studies (4). This result differs from that of earlier animal-based risk models, from which larger human risks were derived (12-13). The model used by Schulz et al differs in 
several important aspects from the previous ones. Frst, adjustment for less-than-lifetime follow-up was more realistic because it took into account increasing CNS cancer rates with age. Second, the exposure assessments developed specifically for each cohort were applied in the model. Third, the CNS cancer potency factor derived from a rat inhalation study (5) was 18 times smaller than the potency factor derived from an ingestion bioassay used for earlier models. The conversion of acrylonitrile potency from animals to humans depends on the exposure metric chosen. Schulz et al (4) used preferably average continuous lifetime exposure rather than lifetime cumulative exposure, the result being lower human risk estimates.

Altogether 24 incident cases of CNS cancer deaths were observed in the 3 cohorts with exposure data as compared with 25.9 expected (4), which corresponds to an SMR of 0.93 [95\% confidence interval (95\% Cl) 0.60 - 1.40]. The prediction derived from the bioassay-based model by Schulz et al (4) was 28.9 deaths, which corresponds to an SMR of 1.11 (95\% C 0.76-1.62). The authors conclude that "The CNS cancer potencies estimated from the best available inhalation bioassay was consistent with the observed deaths in the epidemiologic studies ... [ [p 14]" (4). This is a reasonable conclusion, but it should be added that a lack of association cannot be excluded either. It is extremely difficult to verify or falsify low risk increases for rare diseases in occupational cohort studies. The observed number of deaths from CNS cancer in the 3 cohort studies has to increase more than 10-fold before the lower $95 \%$ C limit for an SMR of 1.11 exceeds unity. Therefore, it is not realistic that an excess risk for ONS cancer deaths due to occupational acrylonitrile exposure, predicted from the rat inhalation bioassay-model, can ever be evaluated against empirical data from epidemiologic studies.

One limitation of the bioassay model for risk estimation used by Schulz et al (4) is that, for both rats and humans, it is based on airborne exposure levels, and not on biologically effective biomarkers of dose, such as hemoglobin adducts. Adduct studies are also needed to clarify whether genetic polymorphisms for CYP2E1 or epoxide hydrolase modifies the formation and detoxification of cyanoethylene oxide in acrylonitrile-exposed humans.

In conclusion it can be said that results from epidemiology and the bioassay model make it obvious that the excess risk for the acrylonitrile-exposed workers cannot have been large. Decision making and the regulation of exposure to acrylonitrile not only has to rely on cancer epidemiology data, but also on other considerations.

\section{References}

1. International Agency for Research on Cancer (IARC). Re-evaluation of some organic chemicals, hydrazine and hydrogen peroxide (part one). Lyon: IARC, 1999. IARC monographs on the evaluation of carcinogenic risks to humans, vol 71.

2. Doll R (guest editor). The mortality of acrylonitrile workers - new evidence and a review of the old. Scand J Work Environ Health 1998;24 suppl 2:3-82.

3. Marsh GM, Youk AO, Collins JJ. A reevaluation of lung cancer risk in the NCI/NIOSH acrylonitrile cohort study. Scand J Work Environ Health 2001;27:5-13.

4. Schulz MR, Hertz-Picciotta I, Todd L, Ball LM. Reconciling animal and human data in a cancer risk assessment of acrylonitrile. Scand J Work Environ Health 2001;27:14-20.

5. Quast JF, Schuetz DJ, Balmer MF, Gushow TS, Park CN, McKenna MJ. A two year toxicity and oncogenicity study with acrylonitrile following inhalation exposure of rats. Midland (MI): Dow Toxicology Research Laboratory, 1980.

6. Woutersen RA. Toxicological profile of acrylonitrile. Scand J Work Environ Health 1998;24 suppl 2:5-9.

7. Lawrence RM, Sweetman GMA, Tavares R, Farmer PB. Synthesis and characterization of peptide adducts for use in monitoring human exposure to acrylonitrile and ethylene oxide. Teratogenesis Carcinog Mutagen 1996;16:139—48.

8. Blair A, Stewart PA, Zaebst DD, Pottern L, Zey JN, Bloom TF, et al. Mortality of industrial workers exposed to acrylonitrile. Scand J Work Environ Health 1998;24 suppl 2:25-41.

9. Wood SM, Buffler PA, Burau K, Krivanek N. Mortality and morbidity of workers exposed to acrylonitrile in fiber production. Scand J Work Environ Health 1998;24 suppl 2:54-62. 
10. Swaen GMH, Bloemen LJN, Twisk J, Scheffers T, Slangen JJM, Collins JJ, et al. Mortality update of workers exposed to acrylonitrile in The Netherlands. Scand J Work Environ Health 1998;24 suppl 2:10—16.

11. Collins JJ, Acquavella JF. Review and meta-analysis of studies of acrylonitrile workers. Scand J Work Environ Health 1998;24 suppl 2:71-80.

12. Ward CE, Starr TB. Comparison of cancer risks projected from animal bioassays to epidemiologic studies of acrylonitrile-exposed workers. Regul Toxicol Pharmacol 1993;18:214-32.

13. Collins JJ, Strother DE. CNS tumors and exposure to acrylonitrile: Inconsistency between experimental and epidemiology studies. Neuro Oncology 1999;1:221-30.

\section{Lars Hagmar, MD \\ Department of Occupational and Environmental Medicine \\ Lund University Hospital \\ SE-22185 Lund Sweden \\ [E-mail: lars.hagmar@ymed.lu.se]}


